\title{
Characterization of circulating microparticle-associated CD39 family ecto-nucleotidases in human plasma
}

\author{
Z. Gordon Jiang • Yan Wu • Eva Csizmadia • Linda Feldbrügge • Keiichi Enjyoji • \\ John Tigges • Vasilis Toxavidis • Holger Stephan • Christina E. Müller • \\ C. James McKnight • Alan Moss • Simon C. Robson
}

Received: 22 June 2014 / Accepted: 18 August 2014 / Published online: 28 August 2014

(C) Springer Science+Business Media Dordrecht 2014

\begin{abstract}
Phosphohydrolysis of extracellular ATP and ADP is an essential step in purinergic signaling that regulates key pathophysiological processes, such as those linked to inflammation. Classically, this reaction has been known to occur in the pericellular milieu catalyzed by membrane bound cellular
\end{abstract}

\footnotetext{
Z. G. Jiang $(\bowtie) \cdot Y$. Wu $\cdot$ E. Csizmadia $\cdot$ L. Feldbrügge $\cdot$

K. Enjyoji $\cdot$ A. Moss $\cdot$ S. C. Robson

Division of Gastroenterology and Hepatology, Department of

Medicine, Beth Israel Deaconess Medical Center, 330 Brookline

Ave, Boston, MA 02115, USA

e-mail: zgjiang@bidmc.harvard.edu

E. Csizmadia $\cdot$ K. Enjyoji $\cdot$ S. C. Robson

Transplant Institute, Beth Israel Deaconess Medical Center, Boston, MA 02115, USA

J. Tigges $\cdot$ V. Toxavidis

Flow Cytometry Core Facility, Beth Israel Deaconess Medical

Center, Boston, MA 02115, USA

Z. G. Jiang $\cdot$ Y. Wu $\cdot$ E. Csizmadia $\cdot$ L. Feldbrügge $\cdot$ K. Enjyoji $・$

J. Tigges $\cdot$ V. Toxavidis $\cdot$ A. Moss $\cdot$ S. C. Robson

Harvard Medical School, Boston, MA 02115, USA

L. Feldbrügge

Department of Surgery, University Hospital Leipzig, Leipzig,

Germany

H. Stephan

Institute of Radiopharmaceutical Cancer Research,

Helmholtz-Zentrum Dresden-Rossendorf, 01314 Dresden, Germany

C. E. Müller

Pharmaceutical Institute, Pharmaceutical Sciences Bonn (PSB), University of Bonn, An der Immenburg 4, 53121 Bonn, Germany

C. J. McKnight

Department of Physiology \& Biophysics, Boston University School of Medicine, Boston, MA 02115, USA
}

ecto-nucleotidases, which can be released in the form of both soluble ecto-enzymes as well as being associated with exosomes. Circulating ecto-nucleoside triphosphate diphosphohydrolase 1 (NTPDase 1/CD39) and adenylate kinase 1 (AK1) activities have been shown to be present in plasma. However, other ecto-nucleotidases have not been characterized in depth. An in vitro ADPase assay was developed to probe the ecto-enzymes responsible for the ectonucleotidase activity in human platelet-free plasma, in combination with various specific biochemical inhibitors. Identities of ecto-nucleotidases were further characterized by chromatography, immunoblotting, and flow cytometry of circulating exosomes. We noted that microparticle-bound ENTPDases and soluble AK1 constitute the highest levels of ecto-nucleotidase activity in human plasma. All four cell membrane expressed E-NTPDases are also found in circulating microparticles in human plasma, inclusive of: CD39, NTPDase 2 (CD39L1), NTPDase 3 (CD39L3), and NTPDase 8. CD39 family members and other ecto-nucleotidases are found on distinct microparticle populations. A significant proportion of the microparticle-associated ecto-nucleotidase activity is sensitive to POM6, inferring the presence of NTPDases, either -2 or/and -3 . We have refined ADPase assays of human plasma from healthy volunteers and have found that CD39, NTPDases 2, 3, and 8 to be associated with circulating microparticles, whereas soluble AK1 is present in human plasma. These ecto-enzymes constitute the bulk circulating ADPase activity, suggesting a broader implication of CD39 family and other ecto-enzymes in the regulation of extracellular nucleotide metabolism.

Keywords Ecto-nucleotidase · CD39 - ATP · ADP . Adenosine $\cdot$ Purinergic signaling $\cdot$ TLC 


\section{Introduction}

Nucleotide release into the extracellular milieu occurs in the setting of cell death, injury, or in the context of channelmediated release, all of which are heightened during inflammation [1-3]. Extracellular adenine nucleotides serve as signal mediators for inflammation, coagulation, vascular tone, and other physiological or pathological processes via type 2 purinergic (P2) receptors $[3,4]$. The catabolism of extracellular adenine nucleotides by ecto-enzymes is a crucial step controlling these purinergic signaling-mediated biological processes, which underpins potential clinical significance in disease diagnosis.

Although attempts to characterize circulating adenine nucleotide metabolism dates back half a century, reliable measurements have required the development of isotope-labeled nucleotides and thin layer chromatographic analysis [5, 6]. These ATPase and ADPase activities in the plasma were found to be independent of alkaline phosphatase as levamisole or $\beta$-glycerophosphate, inhibitors for alkaline phosphatase, did not affect them [6]. Yegutkin and colleagues have demonstrated that at least three distinct ecto-enzymes contribute to the plasma ATPDase activity: adenylate kinase 1 (AK1), CD39, and ecto-nucleotide pyrophosphatase (EPP) [7, 8].

Ecto-nucleoside triphosphate diphosphohydralases (ENTPDases) are largely cell surface-located ecto-enzymes that catalyze the conversion of ATP to ADP and ADP to AMP. To date, eight E-NTPDases have been identified in humans, where four of them, NTPDases 1, 2, 3, and 8 are found to be expressed on the plasma membrane [9, 10]. NTPDase $1 / \mathrm{CD} 39$, the prototype of this family, plays a key role in mediating inflammation in disease entities such as acute lung injury [11], inflammatory bowel disease [12, 13], diabetic nephropathy [14], and ischemic reperfusion injury [15]. Originally described as a B lymphocyte activation marker, CD39 is also expressed on endothelial cells and recently identified on circulating microparticles [16]. Microparticles are extracellular membrane vesicles derived from diverse cell types through heterogeneous mechanisms [17]. Levels of microparticles expressing CD39 have been shown to increase in disease states such as acute liver injury and idiopathic pulmonary arterial hypertension [18, 19].

Whether other E-NTPDase family members are present in the plasma has not been examined in depth, NTPDase 2 (CD39 L1), NTPDase 3 (CD39 L3), and NTPDase 8 share significant sequence homology to CD39 and likely maintain similar protein structures (Fig. 1a) [10]. Homology models of NTPDases 2, 3, and 8 show a highly conserved active site but exhibit more variations in the peripheral domains (Fig. 1b). In a manner similar to CD39, membrane bound NTPDases might be released in exosomes or microparticles and enter the circulation.
Herein, we describe the identification of E-NTPDase activities from circulating microparticles as well as the soluble Ak1 ecto-nucleotidase. These ecto-enzymes account for surprisingly high proportions of the circulating ADPase activity in plasma of healthy individuals and may represent new diagnostic and therapeutic targets for a wide variety of disease states.

\section{Methods and reagents}

Human blood samples

Blood samples from 14 healthy human volunteers were collected through standard phlebotomy into citrate tubes. Erythrocytes and leukocytes were collected by centrifugation at $500 \times \mathrm{g}$ for $10 \mathrm{~min}$ at $25^{\circ} \mathrm{C}$, subsequently washed twice with $\times 10$-fold excess volume of standard phosphate-buffered saline (PBS), and then restored to the original volume. The plasma separated from hematocytes was centrifuged again at $2,500 \times \mathrm{g}$ for $10 \mathrm{~min}$ at $25{ }^{\circ} \mathrm{C}$ to obtain platelet-free plasma, which was subsequently stored at $-80{ }^{\circ} \mathrm{C}$ in aliquots. The study has been approved by IRB at Beth Israel Deaconess Medical Center under A.M.

\section{ADPase activity assay by TLC}

Ecto-ADPase activity assays were performed with $200 \mu \mathrm{M}$ ${ }^{14} \mathrm{C}$-ADP (50 mCi/mmol, American Radiolabeled Chemicals, St. Louis, MO) in the presence of $10 \mathrm{mM}$ calcium chloride and $10 \mathrm{mM}$ magnesium chloride. PBS was used to normalize the phosphate concentration in both samples and standards. The inhibitor concentrations were chosen based on prior literature and used typically $\times 10$-fold that of the inhibitory constant $(\mathrm{Ki})$ or concentrations: EHNA $(150 \mu \mathrm{M})$, POM1 $(26 \mu \mathrm{M})$, POM6 (40 $\mu \mathrm{M})$, Ap5A (80 $\mu \mathrm{M})$, levamisole $(450 \mu \mathrm{M})$, sodium orthovanadate $(20 \mu \mathrm{M})$, and sodium glycerophosphate (1 $\mathrm{mM})[6,8,20]$. The polyoxometalates inhibiting either CD39 and NTPDase 3 (POM1) or NTPDases 2 and 3 (POM6) were used in the reaction (Fig. 1c) [20]. Inhibitors were incubated with platelet-free plasma or blood cell samples for $15 \mathrm{~min}$ before the addition of ADP substrate. For quantitation, recombinant potato apyrase (New England Biolabs, Ipswich, MA) at 0, 50, 100, 150, 200, 300, 400, and $1,000 \mathrm{mU} / \mu \mathrm{l}$ (ATPase activity) was included to generate the standard curve. One unit of ATPase activity was defined as the amount of enzyme that catalyzes the conversion of $1 \mu \mathrm{mol}$ of ATP to ADP in $1 \mathrm{~min}$ at $30{ }^{\circ} \mathrm{C}$. The ATPase activity is 12 times that of the ADPase activity per the manufacturer's instructions. A typical ADPase assay for plasma samples was prepared in a volume of $5 \mu$ land incubated at $30{ }^{\circ} \mathrm{C}$ for $2 \mathrm{~h}$. ADPase assays of hematocytes were prepared in a volume of $20 \mu \mathrm{l}$ and incubated on a Thermomixer (Eppendorf) under the same conditions. The reaction was terminated by adding EDTA to $100 \mathrm{mM}$. A total of $1 \mu \mathrm{l}$ sample was loaded onto a 
A

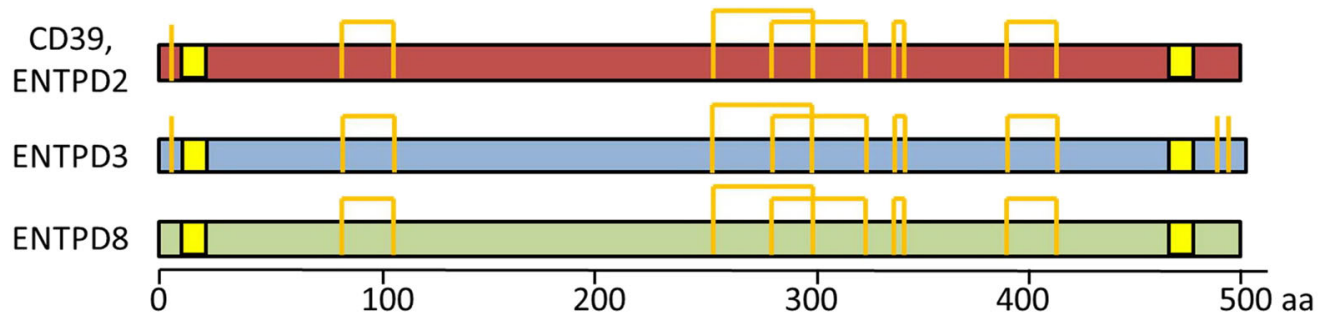

B

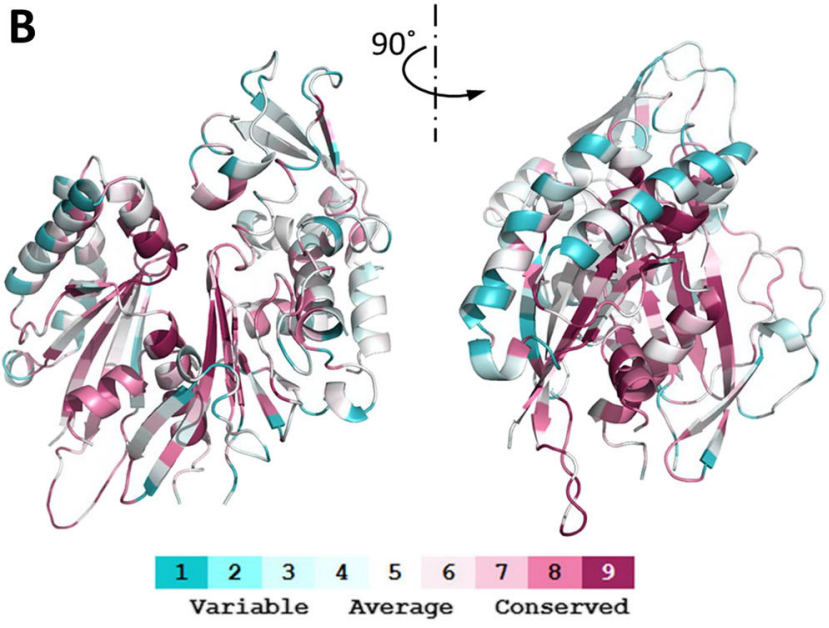

C

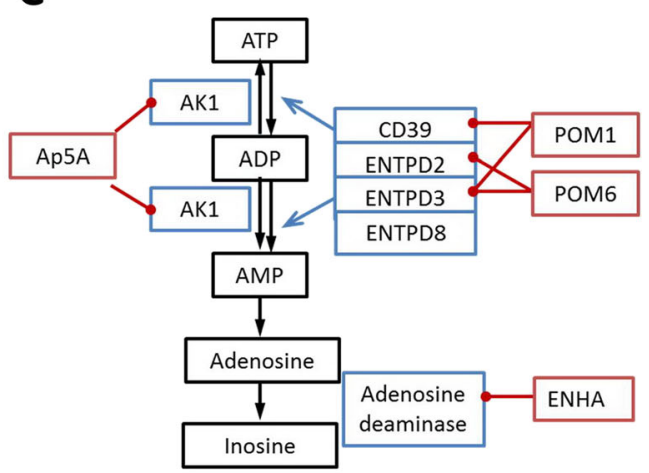

Fig. 1 Structure of E-NTPDases and the ATP catabolism cascade. a Diagram of E-NTPDase primary sequences. The transmembrane domains are highlighted in yellow, cysteine and disulfide bonds are shown by orange lines. b Structure model of human CD39. The homology model is built upon CD39 from Rattus norvegicus (PDB ID 3ZX3). The color

pre-cast silica TLC plate (Sigma-Aldrich, St. Louis, MO). The TLC was performed using $150 \mathrm{ml}$ buffer with isopropanol, ammonia, and water at a ratio of $4: 2: 1$ as previously described [21]. After drying, the isotope-labeled nucleotides were detected by auto-exposure on HyBlot CT films (Denville Scientific, Metuchen, NJ) for 24-72 h. The image was subsequently scanned, converted to 32-bit BW format, and background corrected using ImageJ software (NIH) [22]. The standard curve was fitted with the following sigmoidal function:

$y=\frac{A}{\left(1+10^{\log (B-x) \times C}\right)^{D}}$

where $y$ is ADP conversion (defined by the ratio between adenosine and total nucleotide density), $x$ is ADPase activity in the standards, $A$ is the maximal ADP conversion, $B$ is the $\log$ of inflection point, $C$ is the slope of the transition region, and $D$ is a correction factor for the putative asymmetry of the sigmoidal curve. The ADPase activity in plasma samples was calculated with constants derived from the above equation using a least square deviation regression method. Of note, dilution of samples was made so that measurements fell into the transition region of the curve. The inhibitor specific activity was calculated by subtracting the ADPase activity in the scheme is based on the similarity scores among E-NTPDases 1, 2, 3, 8 as illustrated in the panel. c ATP degradation cascade. Key enzymes involved in the degradation of ATP to inosine are marked in blue boxes with their inhibitors shown in red boxes

presence and absence of inhibitors and reported in both absolute values as well as percentage activity of the total.

\section{Microparticle preparation}

Microparticles were prepared using differential ultracentrifugation as described previously [23]. Briefly, plasma samples were first centrifuged at $10,000 \times g$ for $30 \mathrm{~min}$ at $4{ }^{\circ} \mathrm{C}$. The supernatants were again centrifuged at $100,000 \times \mathrm{g}$ for $90 \mathrm{~min}$ at $4{ }^{\circ} \mathrm{C}$ to collect the microparticles in the pellet. Precipitated microparticles were then re-suspended in PBS for subsequent electrophoresis or flow cytometry analysis.

\section{Size exclusion chromatography}

Five samples were fractionated by size exclusion chromatography using a Superose 6 GL column (GE Healthcare Life Sciences, Pittsburgh, PA) on an ÄKTApurifier (GE Healthcare Life Sciences). Typically, $200 \mu \mathrm{l}$ plasma sample in citrate buffer were centrifuged at $10,000 \times \mathrm{g}$ for $10 \mathrm{~min}$ and injected onto the column. Elution was achieved using TS buffer (20 mM Tris, $150 \mathrm{mM}$ sodium chloride at $\mathrm{pH}$ 7.5) running at $0.5 \mathrm{ml} / \mathrm{min}$ at room temperature. Fractions were collected in $1 \mathrm{ml}$ aliquots through an automated fraction collector. 
Samples from size exclusion chromatography were concentrated using Amicon Ultra filtration units with $5 \mathrm{kDa}$ cutoff (EMD Millipore, Billerica, MA) to the loading volume prior to further analysis.

\section{Electrophoresis and immunoblotting}

Electrophoresis was performed using standard protocols on a pre-cast 4-12\% SDS gradient gel (BioRad Laboratories, Hercules, CA) under non-reducing condition. SDS-PAGE was stained by Coomassie blue or transferred to PVDF membrane for immunoblot analysis. For western blotting, the following antibodies were used: mouse anti-human CD39 (BU61 clone; Ancell Corporation, Bayport, MN), rabbit against human NTPDases 2, 3, and 8 (Thermo Scientific, Rockford, IL). Protein signal was detected using SuperSignal Western Pico Chemiluminescent Substrate (Thermo Scientific) through autoexposure on HyBlot films (Denville Scientific, Metuchen, NJ).

Flow cytometry of microparticles

Annexin V-Pacific Blue (BioLegend, San Diego, CA), monoclonal CD39-FITC (BioLegend), and polyclonal NTPDase 2, 3 , and 8 antibodies labeled with DyLight 488 or $650 \mathrm{~nm}$ Amine-Reactive Dyes (Thermo Scientific) were centrifuged at $10,000 \times g$ at $4{ }^{\circ} \mathrm{C}$ for $15 \mathrm{~min}$ prior to use. Annexin V (BioLegend) was used to label phosphatidylserine and phosphatidylethanolamine, phospholipids that are characteristic for microparticles [24]. The purified microparticles, fluorophorelabeled antibodies, and Annexin V (BioLegend) were incubated in TS buffer with $1 \mathrm{mM}$ calcium chloride in the dark at $4{ }^{\circ} \mathrm{C}$ for $30 \mathrm{~min}$. The products were precipitated again by ultracentrifugation at $100,000 \times g$ for $90 \mathrm{~min}$ at $4{ }^{\circ} \mathrm{C}$, and re-suspended in TS buffer with $1 \mathrm{mM}$ calcium chloride. The flow cytometry experiment was performed on a Gallios Flow Cytometer (Beckman Coulter, Danvers, MA) using standard protocols. One-micromolar sizing beads (Spherotech, Lake Forest, IL) were used as reference to generate gating for microparticles. The data were analyzed using Kaluza (Beckman Coulter).

\section{Protein modeling}

The primary sequences of rat $\mathrm{CD} 39$, human $\mathrm{CD} 39$, and NTPD2, 3, and 8 were downloaded from NCBI protein database and aligned using ClustalW [25]. The threedimensional model of human CD39 was built upon the crystal structure of CD39 from Rattus norvegicus (PDB ID 3ZX3) using SWISS-MODEL [26, 27]. The mapping of conserved protein sequences were obtained via the ConSurf Server [28]. The final model was examined with PyMOL (pymol.org).

\section{Results}

Plasma ADPases demonstrate different inhibitor sensitivity compared to hematocytes

Ecto-nucleotidase activity is known to be present in leukocytes, largely deriving from CD39/NTPDase1, and is therefore expected to be detected in the hematocyte fraction of blood. The nucleotidase activity in the plasma is, however, less well characterized. We first compared the ADPase activity in human hematocytes and platelet-free plasma at physiological $\mathrm{pH}$ (7.4) in the presence of inhibitors specific to different ecto-enzymes. ADP was chosen instead of ATP because of its relative stability and less interference from soluble kinases and EPP (Fig. 1c). As expected, hematocytes demonstrated inhibitor sensitivity primarily to POM1, consistent with the notion that CD39 is the major ecto-nucleotidase on leukocyte membranes (Fig. 2a). In contrast, a distinct inhibitor sensitivity pattern was seen in the plasma. First of all, when ADP was used as a substrate, the catabolism of ADP continued to inosine with no apparent accumulation of AMP and moderate accumulation of adenosine, suggesting the presence of a complex enzyme system that catalyzes the reaction in tandem (Fig. 2b). The ecto-nucleotidase activity was not inhibited by levamisole, orthovanadate, or glycerophosphate, inhibitors for nonspecific phosphatases such as alkaline phosphatase. Instead, plasma ADPase activity was most sensitive to POM6, a non-CD39 inhibitor specific for NTPDases 2 and 3 (Ki 3.9 and $3.8 \mu \mathrm{M}$, respectively) [20] and to a lesser extent to Ap5A (an inhibitor for AK1) (Fig. 2b).

Quantitation of human ADPase activity in the plasma

We then developed an assay to quantitate the ADPase activity in human plasma by incorporating purified recombinant apyrase as an internal standard (Fig. 3a, b). Erythro-9-(2-hydroxy-3-nonyl) adenine (EHNA), an inhibitor for adenylate deaminase 1 , was used in the reaction buffer to inhibit further catabolism of adenosine (Fig. 3c). Human plasma carries an ADPase activity of approximately $30.0 \pm 4.8$ (U/1) (Fig. 3d, Table 1). The presence of POM6, an inhibitor for NTPDases 2 and 3, resulted in the most significant decrease of ADPase activity when compared to Ap5A, an inhibitor for AK1 and POM1, an inhibitor for CD39 and E-NTPDase 3 (Fig. 3d). The mean POM6 sensitive activity in healthy volunteers measured $12.5 \pm 4.8(\mathrm{U} / \mathrm{l})$, representing an average of $42 \%$ of the total ADPase activity in the plasma of healthy volunteers (Table 1). 
A

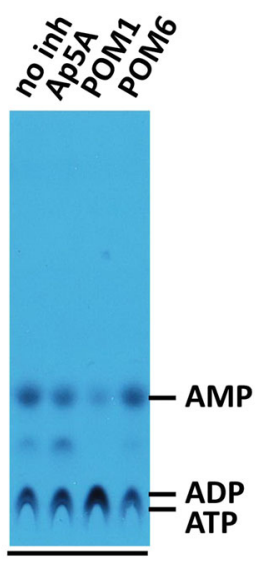

Hematocyte

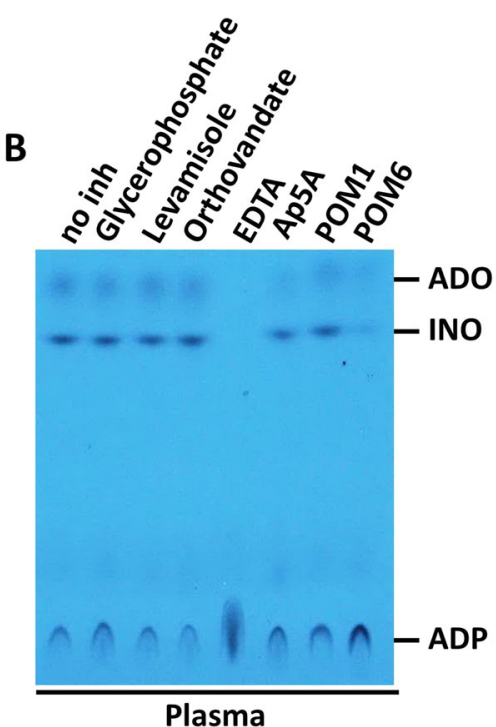

Plasma

Fig. 2 Comparison of ADP catabolism by in human hematocytes and plasma. a ADPase activity of washed hematocytes. b ADPase activity of plasma. ${ }^{14} \mathrm{C}$-ADP is used in the assay and analyzed by TLC as described in the methods. The identities of the nucleotide were determined by ${ }^{14} \mathrm{C}$ nucleotide standards in separate experiments. The inhibitors used are as indicated. Adenosine (ADO) and Inosine (INO) are labeled as indicated

Two types of ADPase activities are present in the plasma

To further delineate ecto-enzymes that gave rise to the ectonucleotidase activity, we fractionated the human plasma on a

Table 1 Human plasma ADPase activity and its inhibitor sensitivity ( $n=$ 14)

\begin{tabular}{lll}
\hline & \multicolumn{2}{l}{ Human ADPase activity (U/L) } \\
\cline { 2 - 3 } & Mean \pm SD & Total (\%) \\
\hline Total & $30.0 \pm 4.8$ & \\
Ap5A sensitive & $6.1 \pm 2.2$ & 20 \\
POM1 sensitive & $4.8 \pm 2.2$ & 16 \\
POM6 sensitive & $12.5 \pm 4.3$ & 42 \\
\hline
\end{tabular}

Superose 6 GL size exclusion column. This step allows the separation of free proteins from much larger circulating particles such as lipoproteins and microparticles (Fig. 4a). The ecto-nucleotidase activity was then measured in each fraction. It became clear that the ecto-nucleotidase activity was present in fraction 1, corresponding to large complexes such as microparticles, and chiefly in fraction 10 , corresponding to free proteins (Fig. 4b). Interestingly, in this experiment, the conversion of ADP was only to AMP, indicating the absence of a 5 '-nucleotidase (5'-NT) like enzymatic activity secondary to size fractionation of the plasma. Thereafter, using a similar inhibitor screening assay as described above, we found that ADPase activity in fraction 1 was sensitive to both POM1 and POM6 suggestive of E-NTPDases (Fig. 4c), whereas the activity in fraction 10 was most sensitive to Ap5 A, consistent with the presence of AK1 (Fig. 4d).
Fig. 3 A plasma ecto-

nucleotidase assay. a ${ }^{14} \mathrm{C}-\mathrm{ADP}$ catalyzed by potato apyrase, an internal standard. b A standard curve of ADP conversion as a function of apyrase concentration as fitted by a sigmoidal function. $\mathrm{c}$ An example of ADPase activity measured in human plasma. The presence of Ap5A, POM1, and POM6 are indicated as above. All lanes contain EHNA that inhibits adenylate deaminase 1 . d Diagram of total ADPase activity in human plasma and the corresponding activity that is sensitive to Ap5A, POM1, and POM6. The error bars represent the standard deviation from twelve human subjects ${ }^{*} p$ value $<0.0001$ )
A

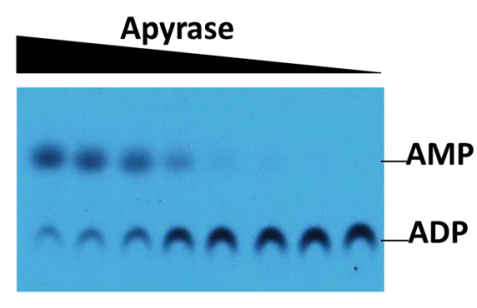

C

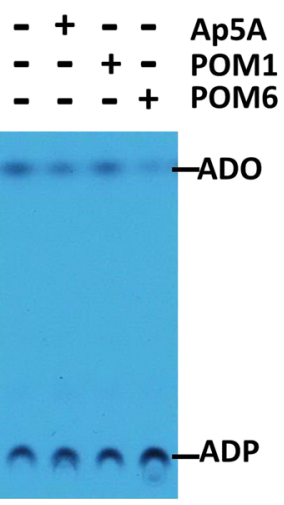

B

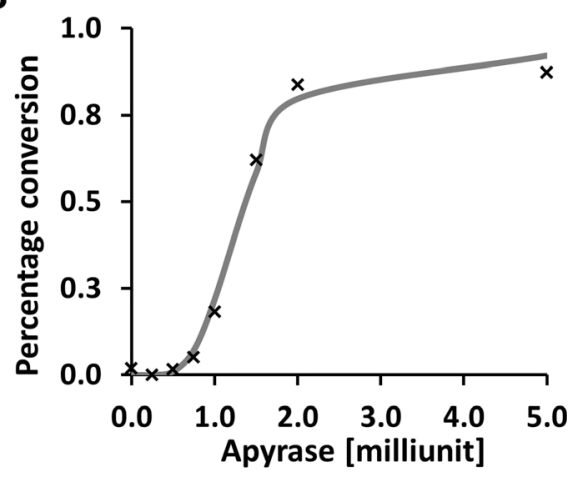

D

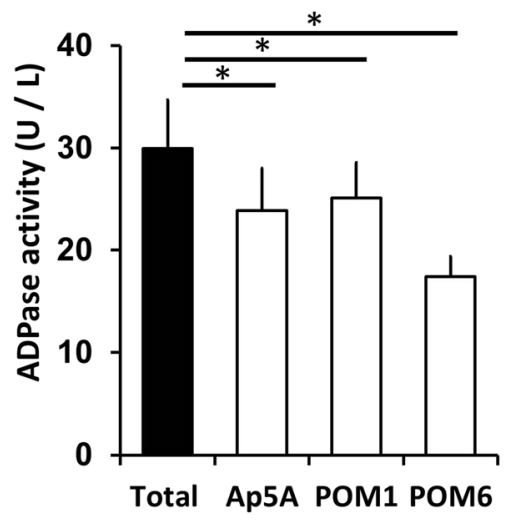


Fig. 4 Characterization of the plasma ecto-nucleotidase activities. a Size exclusion chromatography of human plasma. The chromatogram is shown in red, with collected fractions labeled at the bottom. The molecular weight standards are shown in gray and labeled. b ADPase activity in fractionated human plasma. The ADPase activity sensitive to Ap5A, POM1, and POM6 is further tested in fraction 1 (c) and fraction $10(\mathbf{d})$
A

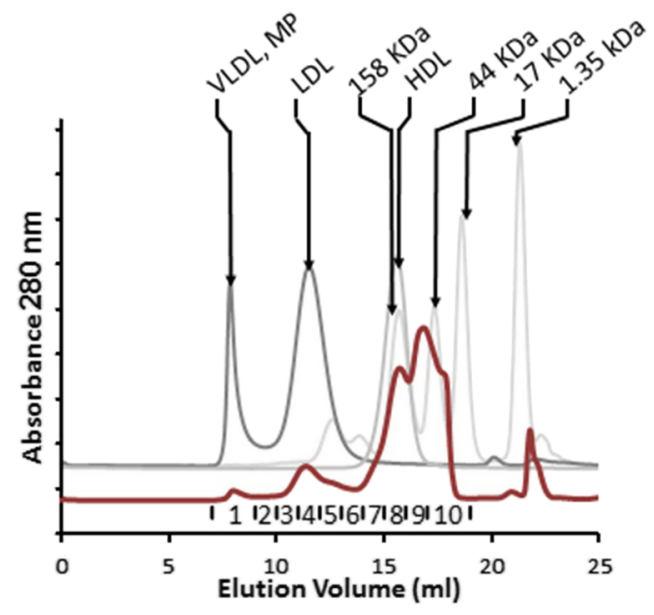

B

$\begin{array}{llllllllll}1 & 2 & 3 & 4 & 5 & 6 & 7 & 8 & 9 & 10\end{array}$
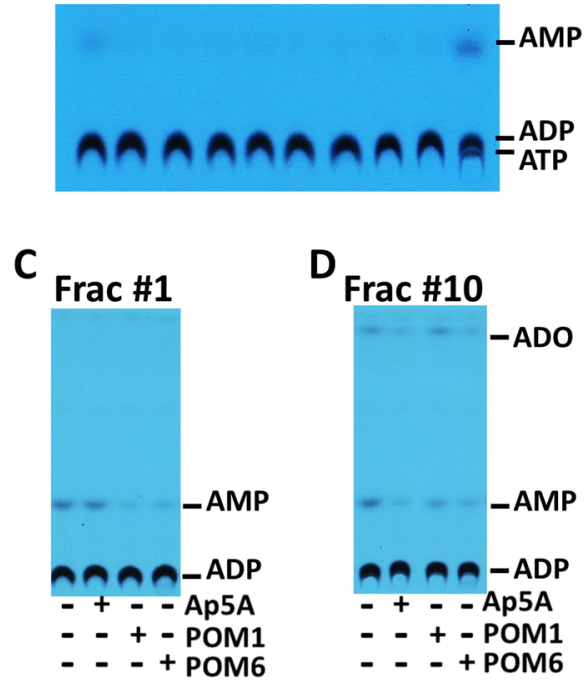

The presence of other E-NTPDases in circulating microparticles

Since POM6 has minimal inhibition of CD39 (Ki> $1 \mathrm{mM}$ ), the presence of POM6 sensitive ADPase activity raised the question whether there are other E-NTPDases in plasma. To test that hypothesis, we performed immunoblotting using antibodies against human CD39, NTPDases 2, 3, and 8 on the human plasma fractionated from the size exclusion column. CD39 as well as NTPDase 2, 3, and 8 were all found in the first fraction corresponding to large complexes such as microparticles (Fig. 5a). The size of the immune-positive bands were close to $100-150 \mathrm{kDa}$ for all four E-NTPDases, suggesting dimers in keeping with previous observations made in CD39 [29, 30].

Next, to confirm the presence of E-NTPDases on circulating microparticles, we isolated microparticles from plasma using differential ultracentrifugation as described previously [23]. Flow cytometry demonstrated the presence of all four members of the CD39 family E-NTPDases in microparticles, albeit the percentage of Annexin $\mathrm{V}^{+} / \mathrm{E}-$ NTPDase $^{+}$particles were significantly lower with NTPDases 3 and 8 (Fig. 6). These data suggest the difference between NTPDases $3^{+}$or $8^{+}$microparticles and $\mathrm{CD} 39^{+}$microparticles. Only polyclonal antibodies are currently available for E-NTPDases other than CD39; these are suboptimal for flow cytometry analysis due to potential nonspecific binding. We, hence, further validated the presence of E-NTPDases by immunoblotting using the same microparticle preparation from three healthy individuals (Fig. 5b). Proteins at expected molecular weights were identified in purified microparticles as the predominant signals on immunoblots with all four antibodies.

\section{Discussion}

The regulation of ATP scavenging and catabolism in the extracellular space is an essential mechanism in

A

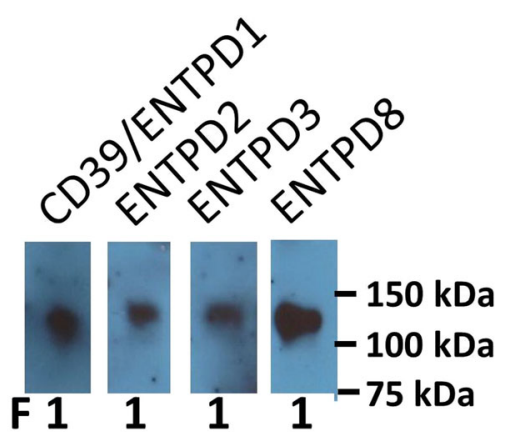

B

\section{3}

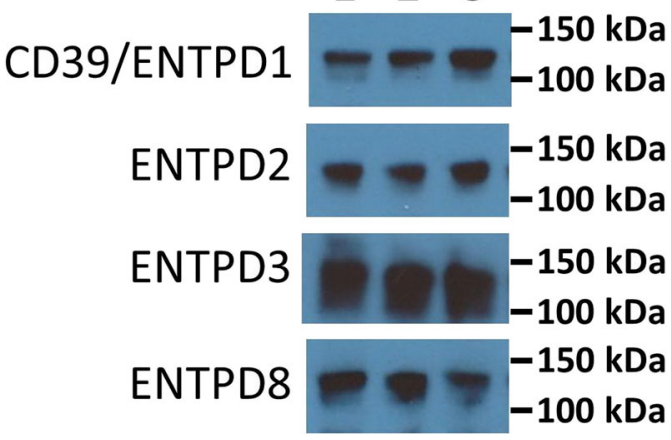

Fig. 5 Immunoblotting of E-NTPDases in fractionated plasma. a Immunoblots of the fractionated plasma using E-NTPDase antibodies. Samples of the first fraction from the size exclusion column were separated by non-reducing SDS-PAGE and then probed with antibodies as indicated. Molecular weight standards are shown in the right lane. b Representative immunoblots of microparticles from human plasma ( 3 of the 14 samples were shown). Microparticles from human subjects were prepared by differential ultracentrifugation and then analyzed by immunoblotting 

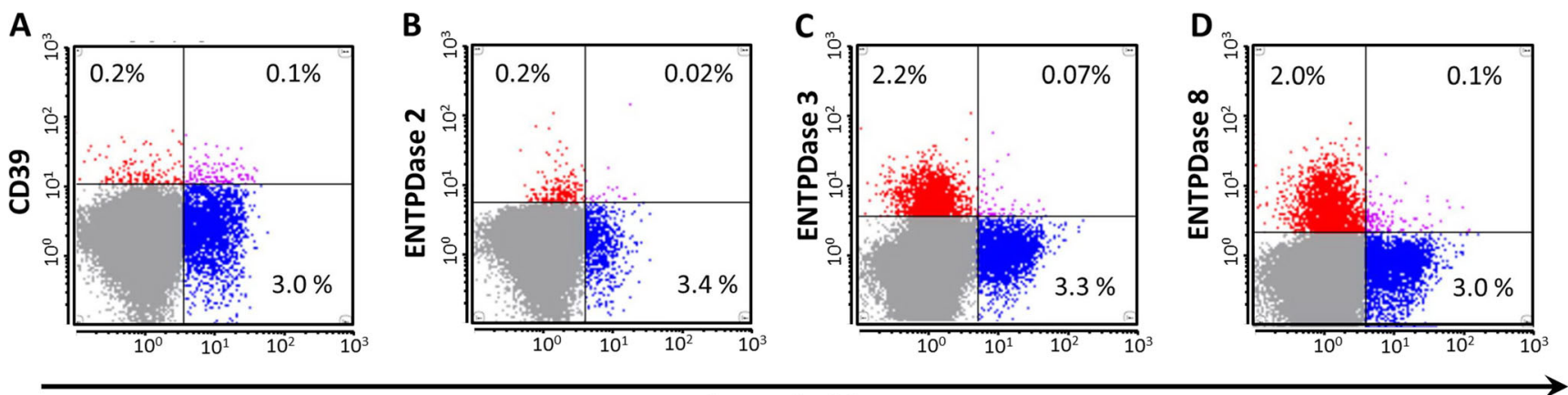

\section{Annexin V}

Fig. 6 Flow cytometry of circulating E-NTPDase-containing microparticles. Representative flow cytometry dot plots are shown with horizontal axis representing Annexin V and vertical axis representing E-NTPDases:
CD39 (a), NTPDase 2 (b), NTPDase 3 (c), and NTPDase 8 (d). The percentages of gated particles $(<1 \mu \mathrm{m})$ are marked in the corresponding quadrants inflammation and is deeply implicated in many diseases. The phosphohydrolysis of extracellular ATP and ADP is believed to occur in a pericellular milieu, catalyzed by cell membrane bound E-NTPDases. This study shows the presence of substantial ecto-nucleotidase activity in human plasma that may complement the nucleotidase activities on the cell surface. We found that at physiological $\mathrm{pH}$, the NTPDases 2,3 , and 8 are present at significant levels on circulating microparticles and contribute to the nucleotidase activities in the human plasma besides the already described AK1 and CD39.

These observations underscore previously underappreciated human physiology with regard to purinergic signaling. E-NTPDase-containing microparticles are present at significant concentrations in healthy volunteer plasma, suggesting that these E-NTPDases are likely expressed at high levels in human tissues that are accessible to circulation. The tissue expression patterns of the genes ENTPD2, 3, and 8 have been reported in the open source proteomics databanks, such as the Human Protein Atlas [31]. Notably, high levels of NTPDase 2 expression have been seen in the adventitia of blood vessels, epithelial cells of the digestive tract, pancreas, epithelial cells of the upper airway, adrenal gland, and cerebral cortex [31, 32]. NTPDase 3 can be seen expressed in various epithelial cells, including kidney, airway, reproductive, and digestive systems, as well as pancreatic islet cells [31]. NTPDase 8 is noted to be abundant in the liver, epithelial cells of the digestive tract, skeletal muscles, and thyroid gland [31]. Compared to ecto-nucleotidases, the physiological significance of AK1 in the plasma is less clear. AK1 is a ubiquitous cytosolic protein most abundantly expressed in muscle cells [33]. Its role is largely linked to intracellular signaling, such as those through AMP sensitive protein kinase, ATP-sensitive potassium channel, and AMP-sensitive metabolic enzymes [34]. It has also been shown that certain myocytes actively secretes AK1 in vitro [35]. The AK1 activity is present in late fractions from the size exclusion column, suggesting its presence as monomer in the plasma. It is unclear whether AK1 in plasma are mainly derived from cytoplasmic leakage or actively secretion.

The critical role of E-NTPDases in immune response has been exemplified by CD39. A recent genome-wide association studies (GWAS) showed that genetic variants of CD39 are the strongest predictor for immunephenotypic traits, surpassing most conventional immunerelated proteins [36]. It is not inconceivable that nonCD39 E-NTPDases are also critical in inflammatory conditions involving the cells where these are highly expressed. For instance, the expression of NTPDases 2, 3 , and 8 in epithelial cells of the digestive tract raises the possibility of their roles in inflammatory diseases of the GI tract. Similarly, in the liver, the respective expression of NTPDase 2, 3, and 8 in portal fibroblasts, activated hepatic stellate cells, and hepatocytes raises their synergistic involvement in liver fibrosis secondary to inflammation $[32,37,38]$. Factors that influence the expression pattern of ecto-enzymes on the surface microparticles remain to be understood. Besides the cell origin, the changes of physiological and pathological states may influence the levels of ecto-enzymes as well as the number of microparticles produced from host cells.

Overall, using the E-NTPDase specific inhibitors, we have developed a refined assay that can differentiate the activities of AK1, and various NTPDases using plasma samples. We have shown the presence of NTPDases 2, 3 , and 8 in circulating microparticles. Together with CD39 and AK1, these ecto-enzymes constitute major ecto-nucleotidase activity in plasma of healthy individuals. Our study indicates a broader presence of CD39 family ecto-nucleotidases participating in purinergic signaling. These ecto-enzymes could be involved in the pathophysiology of inflammatory diseases and provide new targets for disease diagnosis and treatment. 


\section{References}

1. Eltzschig HK, Sitkovsky MV, Robson SC (2012) Purinergic signaling during inflammation. N Engl J Med 367:2322-2333

2. Longhi MS, Robson SC, Bernstein SH, Serra S, Deaglio S (2013) Biological functions of ecto-enzymes in regulating extracellular adenosine levels in neoplastic and inflammatory disease states. $\mathrm{J}$ Mol Med (Berlin) 91:165-172

3. Deaglio S, Robson SC (2011) Ectonucleotidases as regulators of purinergic signaling in thrombosis, inflammation, and immunity. Adv Pharmacol 61:301-332

4. Jacob F, Perez Novo C, Bachert C, Van Crombruggen K (2013) Purinergic signaling in inflammatory cells: P2 receptor expression, functional effects, and modulation of inflammatory responses. Purinergic Signal 9:285-306

5. Jorgensen S (1956) Breakdown of adenine and hypoxanthine nucleotides and nucleosides in human plasma. Acta Pharmacol Toxicol (Copenh) 12:294-302

6. Coade SB, Pearson JD (1989) Metabolism of adenine nucleotides in human blood. Circ Res 65:531-537

7. Yegutkin GG, Samburski SS, Jalkanen S (2003) Soluble purineconverting enzymes circulate in human blood and regulate extracellular ATP level via counteracting pyrophosphatase and phosphotransfer reactions. FASEB J 17:1328-1330

8. Yegutkin GG, Wieringa B, Robson SC, Jalkanen S (2012) Metabolism of circulating ADP in the bloodstream is mediated via integrated actions of soluble adenylate kinase-1 and NTPDase1/ CD39 activities. FASEB J 26:3875-3883

9. Robson SC, Sevigny J, Zimmermann H (2006) The E-NTPDase family of ectonucleotidases: structure function relationships and pathophysiological significance. Purinergic Signal 2:409-430

10. Zimmermann H, Zebisch M, Strater N (2012) Cellular function and molecular structure of ecto-nucleotidases. Purinergic Signal 8:437502

11. Eckle T, Fullbier L, Wehrmann M, Khoury J, Mittelbronn M et al (2007) Identification of ectonucleotidases CD39 and CD73 in innate protection during acute lung injury. J Immunol 178:8127-8137

12. Colgan SP, Eltzschig HK (2012) Adenosine and hypoxia-inducible factor signaling in intestinal injury and recovery. Annu Rev Physiol $74: 153-175$

13. Friedman DJ, Kunzli BM, YI AR, Sevigny J, Berberat PO et al (2009) From the cover: CD39 deletion exacerbates experimental murine colitis and human polymorphisms increase susceptibility to inflammatory bowel disease. Proc Natl Acad Sci U S A 106:1678816793

14. Friedman DJ, Talbert ME, Bowden DW, Freedman BI, Mukanya Y et al (2009) Functional ENTPD1 polymorphisms in African Americans with diabetes and end-stage renal disease. Diabetes 58: 999-1006

15. Eltzschig HK, Eckle T (2011) Ischemia and reperfusion-from mechanism to translation. Nat Med 17:1391-1401

16. Banz Y, Beldi G, Wu Y, Atkinson B, Usheva A et al (2008) CD39 is incorporated into plasma microparticles where it maintains functional properties and impacts endothelial activation. Br J Haematol 142: $627-637$

17. ELA S, Mager I, Breakefield XO, Wood MJ (2013) Extracellular vesicles: biology and emerging therapeutic opportunities. Nat Rev Drug Discov 12:347-357

18. Schmelzle M, Splith K, Andersen LW, Kornek M, Schuppan D et al (2013) Increased plasma levels of microparticles expressing CD39 and CD133 in acute liver injury. Transplantation 95:63-69

19. Visovatti SH, Hyman MC, Bouis D, Neubig R, McLaughlin VV et al (2012) Increased CD39 nucleotidase activity on microparticles from patients with idiopathic pulmonary arterial hypertension. PLoS One 7:e40829

20. Muller CE, Iqbal J, Baqi Y, Zimmermann H, Rollich A et al (2006) Polyoxometalates - a new class of potent ecto-nucleoside triphosphate diphosphohydrolase (NTPDase) inhibitors. Bioorg Med Chem Lett 16:5943-5947

21. Wu Y, Sun X, Kaczmarek E, Dwyer KM, Bianchi E et al (2006) RanBPM associates with CD39 and modulates ecto-nucleotidase activity. Biochem J 396:23-30

22. Schneider CA, Rasband WS, Eliceiri KW (2012) NIH Image to ImageJ: 25 years of image analysis. Nat Methods 9:671-675

23. Kornek M, Popov Y, Libermann TA, Afdhal NH, Schuppan D (2011) Human $\mathrm{T}$ cell microparticles circulate in blood of hepatitis patients and induce fibrolytic activation of hepatic stellate cells. Hepatology 53:230-242

24. Yuana Y, Bertina RM, Osanto S (2011) Pre-analytical and analytical issues in the analysis of blood microparticles. Thromb Haemost 105: 396-408

25. Thompson JD, Higgins DG, Gibson TJ (1994) CLUSTAL W: improving the sensitivity of progressive multiple sequence alignment through sequence weighting, position-specific gap penalties and weight matrix choice. Nucleic Acids Res 22:4673-4680

26. Arnold K, Bordoli L, Kopp J, Schwede T (2006) The SWISSMODEL workspace: a web-based environment for protein structure homology modelling. Bioinformatics 22:195-201

27. Zebisch M, Krauss M, Schafer P, Strater N (2012) Crystallographic evidence for a domain motion in rat nucleoside triphosphate diphosphohydrolase (NTPDase) 1. J Mol Biol 415:288-306

28. Glaser F, Pupko T, Paz I, Bell RE, Bechor-Shental D et al (2003) ConSurf: identification of functional regions in proteins by surfacemapping of phylogenetic information. Bioinformatics 19:163-164

29. Stout JG, Kirley TL (1996) Control of cell membrane ecto-ATPase by oligomerization state: intermolecular cross-linking modulates ATPase activity. Biochemistry 35:8289-8298

30. Schulte am Esch J 2nd, Sevigny J, Kaczmarek E, Siegel JB, Imai M et al (1999) Structural elements and limited proteolysis of CD39 influence ATP diphosphohydrolase activity. Biochemistry 38:22482258

31. Uhlen M, Oksvold P, Fagerberg L, Lundberg E, Jonasson K et al (2010) Towards a knowledge-based Human Protein Atlas. Nat Biotechnol 28:1248-1250

32. Fausther M, Lecka J, Soliman E, Kauffenstein G, Pelletier J et al (2012) Coexpression of ecto-5'-nucleotidase/CD73 with specific NTPDases differentially regulates adenosine formation in the rat liver. Am J Physiol Gastrointest Liver Physiol 302: G447-459

33. Panayiotou C, Solaroli N, Karlsson A (2014) The many isoforms of human adenylate kinases. Int J Biochem Cell Biol 49C:75-83

34. Dzeja PP, Chung S, Terzic A (2007) In: Saks V (ed) Molecular system bioenergetics. Wiley-VCH, Weinheim

35. Choo HJ, Kim BW, Kwon OB, Lee CS, Choi JS et al (2008) Secretion of adenylate kinase 1 is required for extracellular ATP synthesis in C2C12 myotubes. Exp Mol Med 40:220-228

36. Orru V, Steri M, Sole G, Sidore C, Virdis F et al (2013) Genetic variants regulating immune cell levels in health and disease. Cell 155: $242-256$

37. Andrade CM, Wink MR, Margis R, Borojevic R, Battastini AM et al (2010) Changes in E-NTPDase 3 expression and extracellular nucleotide hydrolysis during the myofibroblast/lipocyte differentiation. Mol Cell Biochem 339:79-87

38. Fausther M, Lecka J, Kukulski F, Levesque SA, Pelletier J et al (2007) Cloning, purification, and identification of the liver canalicular ecto-ATPase as NTPDase8. Am J Physiol Gastrointest Liver Physiol 292:G785-795 